\title{
Back Handspring Robot - Target Dynamics-Based Control
}

\author{
Sang-Ho Hyon \\ Dept. of Bioengineering \& Robotics \\ Tohoku University \\ Sendai 980-8579, Japan \\ Email: sangho@ieee.org \\ Naoto Yokoyama \\ Dept. of Bioengineering. \& Robotics \\ Tohoku University \\ Sendai 980-8579, Japan
}

\author{
Takashi Emura \\ Dept. of Bioengineering \& Robotics \\ Tohoku University \\ Sendai 980-8579, Japan \\ Email: emura@emura.mech.tohoku.ac.jp
}

\begin{abstract}
This paper reports on a gymnastic robot, which are developed for various floor exercises such as jumping, somersault and back handspring. The robot is a planar and serially connected four-link robot, whose joints are fully actuated by electric servomotors. In this paper, the modeling and the controller for back handspring are addressed. The controller is derived from task-specific target dynamics and its model matching. The use of global physical quantities such as center of mass, or angular momentum allows even simple target dynamics to generate complex gymnastic motions of multi-body system. The effectiveness of the controller is confirmed via simulations and experiments.
\end{abstract}

\section{INTRODUCTION}

Realization of complex and fast motion task is important and attractive area of research. It enhances the mobility of humanoid robots and also contributes to good understanding of motor control of human. Gymnastic motion is included in such interesting motion tasks. This paper reports on a gymnastic robot, which can perform jumping, somersault, and back handspring (Fig. 1).

There are some related studies on gymnastic robots. Raibert and his co-workers succeeded in jumping and somersault control of a 3D biped robot [1] [2]. Since the robot has telescopic leg, jumping or flipping is rather easy to realize by some intuitive controllers. However, it becomes impossible to derive such a intuitive controllers when the robot has articulated multi-link structure and highly nonlinear dynamics. On the other hand, Arikawa succeeded in jumping control of five-link robot by solving numerically two-point boundary problem to obtain joint trajectories and applying high-gain local feedback control to each joint [3].

When human performs gymnastic motion, learning and its feed-forward control may plays dominant role. However, as long as the learning is based on sensory information, feedback controllers can give important insights into the learning mechanism, especially when the motion is generated only by feedback controller. Moreover, feedback controllers have their own advantages over feed-forward ones; a robustness against sudden change of environment is the one of them. In this context, there are some successful examples of high bar robot by using real-time feedback controllers. Spong and Yamakita realized swing up and balancing control [4][5]. Nakanishi succeeded in brachiating control of a two-link robot [6]. Recently Yamakita

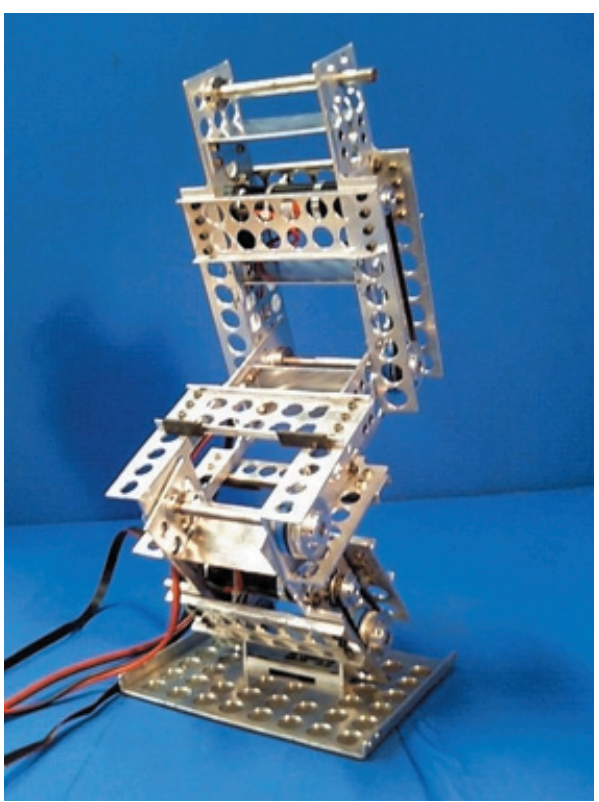

Fig. 1. Four-link planar gymnastic robot

demonstrated continuous motion of swingup $\rightarrow$ giant $\rightarrow$ somersault $\rightarrow$ landing $\rightarrow$ balancing, by multi-link robot [7]. Nonlinear feedback control is also effective to jumping and flipping motion. For example, Berkemeier applied feedback linearization technique to underactuated robot (foot can freely rotate like a pivot joint) [8]. They found zero-dynamics, which result from partial feedback linearization, to produce periodic hopping or flipping motion. The same approach could be found in [9].

The objective of this paper is to propose a new control framework of gymnastic robots that performs floor exercise. To do so, here we consider a full-actuated planar model. The robot has four degrees of freedom (DOF), which can describe "global dynamics" of planar gymnastic motions as described later. From the theoretic point of view, underactuated robots and their controllers are of interest. However, to realize more complex and fast gymnastic motions, we need "fully actuated" model. "Fully actuated" means the robot has a foot connected with actuated ankle joint. Ankle torque can provide a large angular momentum of the total system around the contact point, which is 
TABLE I

ROBOT PARAMETERS

\begin{tabular}{c|c|c|c|c}
\hline & Link0 & Link1 & Link2 & Link3 \\
\hline Mass [kg] & 0.135 & 0.626 & 0.533 & 0.579 \\
\hline Link length [m] & 0.09 & 0.13 & 0.15 & 0.19 \\
\hline *Position of C.M. [m] & 0.074 & 0.031 & 0.55 & 0.086 \\
\hline Inertia [kgmm ${ }^{2}$ ] & 260 & 1200 & 880 & 1600 \\
\hline
\end{tabular}

*Position of C.M. means the distance between C.M. of each link and their proximal joint. All principal axes of each link are nearly coincident with their center axes.

critical to gymnastic motion associated with high speed rotation. It can also be used for balancing task.

Our control strategy is to impose some target dynamics that describe gymnastic motions and realize them by model matching controller. We expect the use of global physical quantities such as center of mass, or angular momentum allow even simple target dynamics to generate complex gymnastic motions of multi-body system.

\section{RoBOT MODEL}

\section{A. Mechanical model development}

Figure 1 shows a newly developed planar gymnastic robot. The robot has four links, serially connected by three joints. Each joint is actuated by geared servomotor (Maxon $20 \mathrm{~W}$ ) through a timing belt. The total reduction ratio is 10.8. Most of the mechanical parts are machined out of aluminum frame. Overall height of the robot is $0.46 \mathrm{~m}$ and the total weight is about $1.74 \mathrm{~kg}$. Physical parameters are summarized in Table 1.

The controller is implemented and runs in real time on a Linux PC (PentiumIII 500MHz). The control period is $1.3 \mathrm{~ms}$. Servomotors are driven by DC servo-drivers with the torque control mode. Commanded signal to the servodriver is fed by DA converter and joint angles are obtained by the encoders mounted to servomotors via digital I/O. All the interface is provided by a ISA-bus interface board. The robot does not carry the computer and servo-drivers because it is at prototype stage. Also, gyro is not installed because the motion is too fast. To detect the ground contact of the robot, touch switches are attached to the floor.

\section{B. Mathematical model representation}

Figure 2 shows the definition of mathematical model. To derive equations of motion for stance phase and flight phase at once, generalized coordinates includes absolute position. That is, the generalized coordinates are defined as the position of the toe, $\left(x_{0}, z_{0}\right)$, and the angles, $\psi=$ $\left(\psi_{0}, \psi_{1}, \psi_{2}, \psi_{3}\right)$, where $\psi_{0}$ is the attitude of the link 0 (foot), and $\psi_{1}, \psi_{2}$ and $\psi_{3}$ are the angles of joint 1 (ankle), joint 2 (knee) and joint 3 (hip) respectively.

We assume the foot does not bounce back, nor slip the ground (inelastic impulsive impact) This assumption is required to introduce impulse equation. As shown in Fig. 3, a back handspring motion is composed of successive phase transitions; Stance 1 (standing on the feet) $\rightarrow$ Flight 1 $\rightarrow$ Touchdown $2 \rightarrow$ Stance 2 (standing on the head) $\rightarrow$ Touchdown $1 \rightarrow \cdots$. It is similar to the phase transition of running motion. Motions of the touchdown phases are

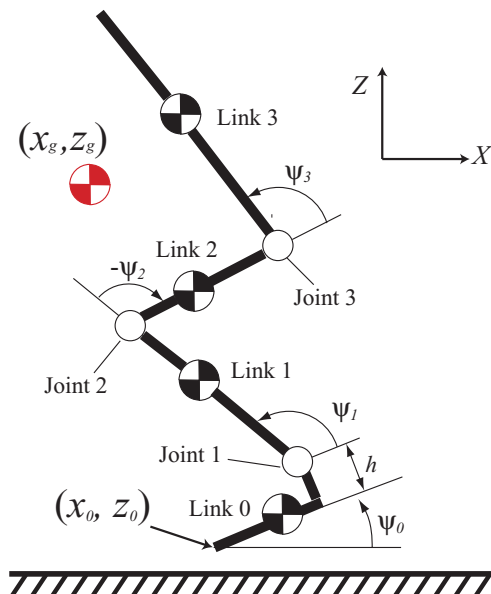

Fig. 2. Definition of mathematical model

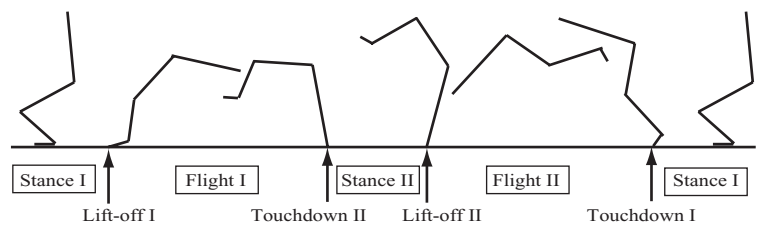

Fig. 3. Phase transition of back handspring

described by impulse equations. When human performs back handspring, thanks to the hyper-multi-link structure, the touchdown is very smooth and no "chattering" occurs between the foots (or hands) and the floor.

We aim to such a smooth touchdown, but do not want to make it complicated unnecessarily. That is why we introduce inelastic impulse assumption. To meet the assumption, a soft mat is covered on the floor.

\section{Equations of motion at Stance I and Flight I}

The equations of motion can be derived by Lagrange's formulation.

$$
\begin{aligned}
J(q) \ddot{q}+H(q, \dot{q}) & =u+E^{T} \lambda \\
E \dot{q} & =0,
\end{aligned}
$$

where $q=\left(x_{0}, z_{0}, \psi\right) \in R^{6}$ is the generalized coordinates, $J(q) \in R^{6 \times 6}$ is a inertial matrix and $H(q, \dot{q}) \in R^{6}$ is a nonlinear term. The nonlinear term includes joint-wise viscous friction, which is identified by experiments. The generalized force $u \in R^{6 \times 1}$ contains joint torques $\tau=$ $\left(\tau_{1}, \tau_{2}, \tau_{3}\right)^{T} \in R^{3}$. Matrix $E:=\frac{\partial \Phi_{0,1}(q)}{\partial q}$ represents the derivative of the constraint for the position of the toe and for the posture the foot:

$$
\Phi_{0}(q)=\left[\begin{array}{c}
x_{0} \\
z_{0} \\
\psi_{0}
\end{array}\right]=0,
$$

or, only for the position of the toe:

$$
\Phi_{1}(q)=\left[\begin{array}{l}
x_{0} \\
z_{0}
\end{array}\right]=0 .
$$


The associated constraint force is represented by $\lambda_{0}:=$ $\left(\lambda_{x}, \lambda_{z}\right)^{T} \in R^{2}$ or $\lambda_{1}:=\left(\lambda_{x}, \lambda_{z}, \lambda_{\psi}\right)^{T} \in R^{3}$. They can be calculated as:

$$
\lambda=-\left(E J^{-1} E^{T}\right)^{-1} E J^{-1}(u-H) .
$$

At the beginning, the robot keeps Eq. (3). If the zero crossing of $\lambda_{\psi}$ is detected, the heel is off the ground and the constraint switches to (4). Dynamics of flight phase can be obtained by removing $\lambda$ from (1).

We will further apply coordinate transformation so that we can directly handle the position of the center of mass (C.M.) of total system.

Using new generalized coordinates $q_{g}=\left(x_{g}, z_{g}, \psi\right)$, Eq. (1) is transformed to:

$$
\begin{aligned}
J_{g}\left(q_{g}\right) \ddot{q}_{g}+H_{g}\left(q_{g}, \dot{q}_{g}\right) & =u+E_{g}\left(q_{g}\right)^{T} \lambda_{g} \\
E_{g}\left(q_{g}\right) \dot{q}_{g} & =0 .
\end{aligned}
$$

Note that (7) is a moving constraint because of the coordinate transformation (compare with (2)). Threfore, the constraint force becomes:

$$
\lambda_{g}=-\left(E_{g} J_{g}^{-1} E_{g}^{T}\right)^{-1}\left(\gamma+E_{g} J_{g}^{-1}\left(u-H_{g}\right)\right),
$$

where $\gamma=\frac{\partial}{\partial q_{g}}\left(E_{g}\left(q_{g}\right) \dot{q}_{g}\right) \dot{q}_{g}$.

Equation (6) has a decomposed structure of translational motion and rotary motion as shown below.

$$
\begin{aligned}
M \ddot{x}_{g} & =\lambda_{x} \\
M \ddot{z}_{g} & =-M g+\lambda_{z} \\
J_{\psi}(\psi) \ddot{\psi}+H_{\psi}(\psi, \dot{\psi}) & =\left[\begin{array}{l}
0 \\
\tau
\end{array}\right]-\frac{1}{m} R(\psi) \lambda_{\psi}
\end{aligned}
$$

Here, $M=m_{1}+m_{2}+m_{3}$ is the total mass, $g$ is gravity acceleration, and $\mathrm{R}$ is a transformation matrix. $J_{\psi} \in R^{4 \times 4}$ and $H_{\psi} \in R^{4}$ are the inertia matrix and the nonlinear terms respectively, which are related only to $\psi$ or $\dot{\psi}$.

\section{Equations of motion at Touchdown I}

Touchdown equation can be expressed as Lagrange's impulsive equation, which can be found in some classical dynamics textbook [10].

$$
\left[\begin{array}{cc|c}
M & 0 & 0_{2 \times 4} \\
0 & M & \\
\hline 0_{4 \times 2} & J_{\psi}
\end{array}\right]\left[\begin{array}{c}
\Delta \dot{x_{g}} \\
\Delta \dot{z}_{g} \\
\hline \Delta \dot{\psi}
\end{array}\right]=E_{g}^{T} \hat{\lambda},
$$

where $\Delta \dot{x}_{g}:=\dot{x}_{g+}-\dot{x}_{g-}, \Delta \dot{z}_{g}:=\dot{z}_{g+}-\dot{z}_{g-}$ and $\Delta \dot{\psi}:=$ $\dot{\psi}_{+}-\dot{\psi}_{-}$are the instantaneous velocity changes just before and after touchdown, and $\hat{\lambda} \in R^{2}$ is the constraint impulse associated with the instantaneous velocity constraint at touchdown:

$$
E_{g}\left[\begin{array}{c}
\dot{x}_{g+} \\
\dot{z}_{g+} \\
\dot{\psi}_{+}
\end{array}\right]=0
$$

Combining (12) and (13), we can calculate $\hat{\lambda}, \dot{x}_{g+}, \dot{z}_{g+}$, and $\dot{\psi}_{+}$for given variables just before the impact.

Equations for Stance II, Flight II and Touchdown II can be easily obtained by swapping coordinates.

\section{BACK HANDSPRING CONTROLLER}

\section{A. Target dynamics}

Back handspring is a rapid and complex motion task composed of multiple phases. In the control problem, it is not realistic to depend on some pre-planned reference trajectories, because in general such a trajectory-tracking scheme cannot adapt to the changes of environment, which are difficult to predict. Instead, we try to achieve complex motion tasks by describing simple target dynamics (equation of motion) about global physical quantities such as C.M. or momentum. The same approach could be found in control of running robots (e.g. [11]).

1) Stance $I$

At Stance I with the heel supporting, target dynamics is designed as:

$$
\begin{aligned}
\ddot{\ddot{x}} & =a \\
M \ddot{\bar{z}} & =-K_{z}\left(\bar{z}-z_{e 1}\right) \\
\dot{\bar{P}}_{g} & =-K_{p}\left(\bar{P}_{g}-P_{g d}\right),
\end{aligned}
$$

where the "barred" variables implies those of target dynamics to be realized in Section 3.2. The first part is a horizontal dynamics of constant acceleration, the second part is simple oscillation around $z_{e 1}$, and the last part is to control $P_{g}$, the angular momentum around C.M., to a desired value $P_{g d}$.

When the heel leaves the ground, the robot stands on its toe. There is no available applied torque around contact point: the system becomes to underactuated. In particular, we cannot control $P_{g}$ arbitrarily in this phase. Therefore we abandon Eq. (16) by turning off the ankle torque, $\tau_{1}=0$. 2) Flight I

At Flight I, there are no target dynamics designed, for this time. Instead, joint angles are locally controlled to a specified target configuration $\left(\psi_{d}=\psi_{1 d}, \psi_{2 d}, \psi_{3 d}\right)$ until the top of the link 4 (head) touches down. The configuration is empirically determined. Here, touchdown point is the most important because at the subsequent phase, Stance II, there is no available applied torque around contact point as the same as Stance I with toe supporting. Touchdown point affects much on the rotational speed (horizontal speed) of the robot. We will pose this problem to the future work and do not discuss here.

3) Stance II

At Stance II, target dynamics is set only to C.M.:

$$
\begin{aligned}
\ddot{\ddot{x}} & =a_{2} \\
M \ddot{\bar{z}} & =-K_{z}\left(\bar{z}-z_{e 2}\right) .
\end{aligned}
$$

\section{4) Flight II}

Controller at Flight II is the same as Flight I, except that $\psi_{d}$ is empirically determined to stop the motion of the robot.

\section{B. Model matching control}

This section derives control input $\tau$ to realize the above target dynamics. First, remember Eq. (9) and (10). They say that we can control $\ddot{x}_{g}$ and $\ddot{z}_{g}$ arbitrarily via $\lambda_{x}$ and $\lambda_{z}$, 
which are nothing but ground reaction forces. Substituting (14) and (15) to (9) and (10), it seems we can solve $\tau$ from Eq. (8).

Rearranging (8) yield the following equations.

$$
\begin{aligned}
& \underbrace{E_{g} J_{g}^{-1}}_{A_{1}}\left(u-H_{g}\right)=\underbrace{\gamma-\left(E_{g} J_{g} E_{g}^{T}\right) \lambda_{g}}_{B_{1}} \\
& {\left[\begin{array}{ll}
A_{11} & A_{12}
\end{array}\right]\left(\left[\begin{array}{l}
0 \\
\tau
\end{array}\right]-H_{g}\right)=B_{1}} \\
& A_{12} \tau=B_{1}+A H_{g}
\end{aligned}
$$

Since $A_{12}$ is non-invertible, to determine "uniquely" the control inputs, we need another target dynamics. With the target dynamics about $P_{g}$, we can solve it. $P_{g}$ can be expressed as:

$$
P_{g}=J_{0} \dot{\psi}_{0}+J_{1} \dot{\psi}_{1}+J_{2} \dot{\psi}_{2}+J_{3} \dot{\psi}_{3}
$$

where $J_{i}(i=0,1,2,3)$ is nonlinear inertia terms. Its time derivative is calculated as:

$$
\dot{P}_{g}=J_{0} \ddot{\psi}_{0}+J_{1} \ddot{\psi}_{1}+J_{2} \ddot{\psi}_{2}+J_{3} \ddot{\psi}_{3}+J_{4}
$$

Substituting (16), we get the following equation.

$$
\underbrace{\left[\begin{array}{llll}
J_{0} & J_{1} & J_{2} & J_{3}
\end{array}\right] J_{g}^{-1}}_{A_{2}}\left(u-H_{g}\right)=\underbrace{\dot{\overline{P_{g}}}}_{B_{2}}
$$

Combining (21) and (24) gives

$$
\left[\begin{array}{c}
A_{12} \\
A_{2}
\end{array}\right] \tau=\left[\begin{array}{c}
B_{1} \\
B_{2}
\end{array}\right]+A H_{g} .
$$

This time, the matrix of the left hand side is invertible and we can obtain control input $\tau$.

\section{Simulation AND EXPERIMENT}

Parameters to be determined are;

- Initial configuration $\psi(0)$

- $a_{1}, K_{z}, z_{e 1}, P_{g d}, a_{2}, z_{e 2}$ (Stance I and Stance II)

- Desired touchdown posture $\psi_{d}$ (Flight I and Flight II) Moreover, we should consider about torque limits. In our case, it is about $\pm 1.5 \mathrm{Nm}$.

These parameters are roughly tuned step by step along to the following way. First $\psi(0)$ is determined from $x_{g}(0)$ and $z_{g}(0) . x_{g}(0)$ is just above the ankle. Then, $z_{g}(0), a_{1}, K_{z}$, $z_{e 1}$ and $P_{g d}$ in Stance I are tuned so that the head touches down as fast as possible. Doing so, the flight time becomes short. Therefore, we can choose $\psi_{d}$ as the terminal position of Stance I. If the angular momentum $P_{g}$ has enough level, it is easy for the robot to rotate around its pivot (head) in Stance II. We first try to set $P_{g d}$ to some constant value to be reached, but the results are not satisfactory; the robot rotates too fast than the translational motion of $\left(x_{g}, z_{g}\right)$. Therefore, we modify it by

$$
P_{g d}=-c\left(x_{g}-x_{g}(0)\right),
$$

where $c$ is a constant. This comes from our observation that gymnast increases his rotational speed when C.M. proceeds enough.

The parameters in Stance II are difficult to choose. The difficulty comes from the underactuated structure of Stance
II: $P_{g}$ is not controlled in this phase. This implies we cannot expect enough $P_{g}$ at the second lift-off. Therefore, we set $z_{e 2}$ and $K_{z 2}$ larger than those of Stance I to make the robot take off higher. The higher the robot jumps, the more flight time for the controlling final configuration is given, even if $P_{g}$ is not enough. As in Stance I, admissible region of these parameters is rather limited because they strongly affect on the posture of the robot. The final parameter $\psi_{d}$ in Flight II is tuned so that the robot can stop and standstill. In simulation we can control the robot to standstill in the next Stance I, but in experiment the robot is controlled to have some final posture, which is unchanged until it stops.

One of the parameter set thus determined is shown in Table 2. Using these parameters, successful back handspring motion is performed in both simulation and experiment. Fig. 4 depicts the animation created by simulation. Snap shots of corresponding experiment are shown in Fig. 5. Since the robot is not installed with high-speed gyro (it requires more than $1000 \mathrm{deg} / \mathrm{s} \mathrm{!),} \mathrm{in} \mathrm{the} \mathrm{experiment} \mathrm{the}$ robot is controlled in open-loop. The video of the experiment is included in the conference proceedings. Fig. 6 and Fig. 7 show the joint angles and joint torques, together with ON/OFF state of floor switches $(\mathrm{On}=1$, Off $=0)$ at the simulation and experiment. Each joint torque in the experiment is obtained by motor current via $\mathrm{AD}$ converter.

Although the back handspring finishes within a very short period of time (about $0.6 \mathrm{sec}$ !), these two figures show clearly the time evolution of each variables and phase transitions. Until the first touchdown $(0.3 \mathrm{~s})$, there are no significant differences between the simulation and experiment: model matching controller works perfectly. After the touchdown, however, the differences become larger. The most significant difference is the period of Stance II. The stance period of simulation is about 0.1 $\mathrm{s}$, while that of experiment is about $0.05 \mathrm{~s}$. This implies the difference mainly comes from the modeling error of touchdown phase: we can say the mat covers the floor does not emulate inelastic impulse assumption well. But we are optimistic about this error because we can take other options (e.g. installing dampers to the top of the robot).

\section{CONCLUSION}

We developed a gymnastic robot for various floor exercise such as jumping, somersault and back handspring. The robot is a planar and serially connected four-link robot, whose joints are actuated by electric servomotors. Then we described about our control strategy of fast and complex gymnastic motion, which is different from the classical

TABLE II

CONTROL PARAMETERS

\begin{tabular}{c|c}
\hline Parameter & Value \\
\hline$\psi_{1}(0), \psi_{2}(0), \psi_{3}(0)$ & $120,-94,90$ \\
$a_{1}, z_{e}, K_{z}, c @$ Stance I & $4.5,0.19,20,4.2$ \\
$a_{2}, z_{e 2}, K_{z} @$ Stance II & $3.8,0.56,40$ \\
$\psi_{1 d}, \psi_{2 d}, \psi_{3 d} @$ Flight I & $0,-70,-100$ \\
$\psi_{1 d}, \psi_{2 d}, \psi_{3 d} @$ Flight II & $125,-125,50$ \\
\hline
\end{tabular}



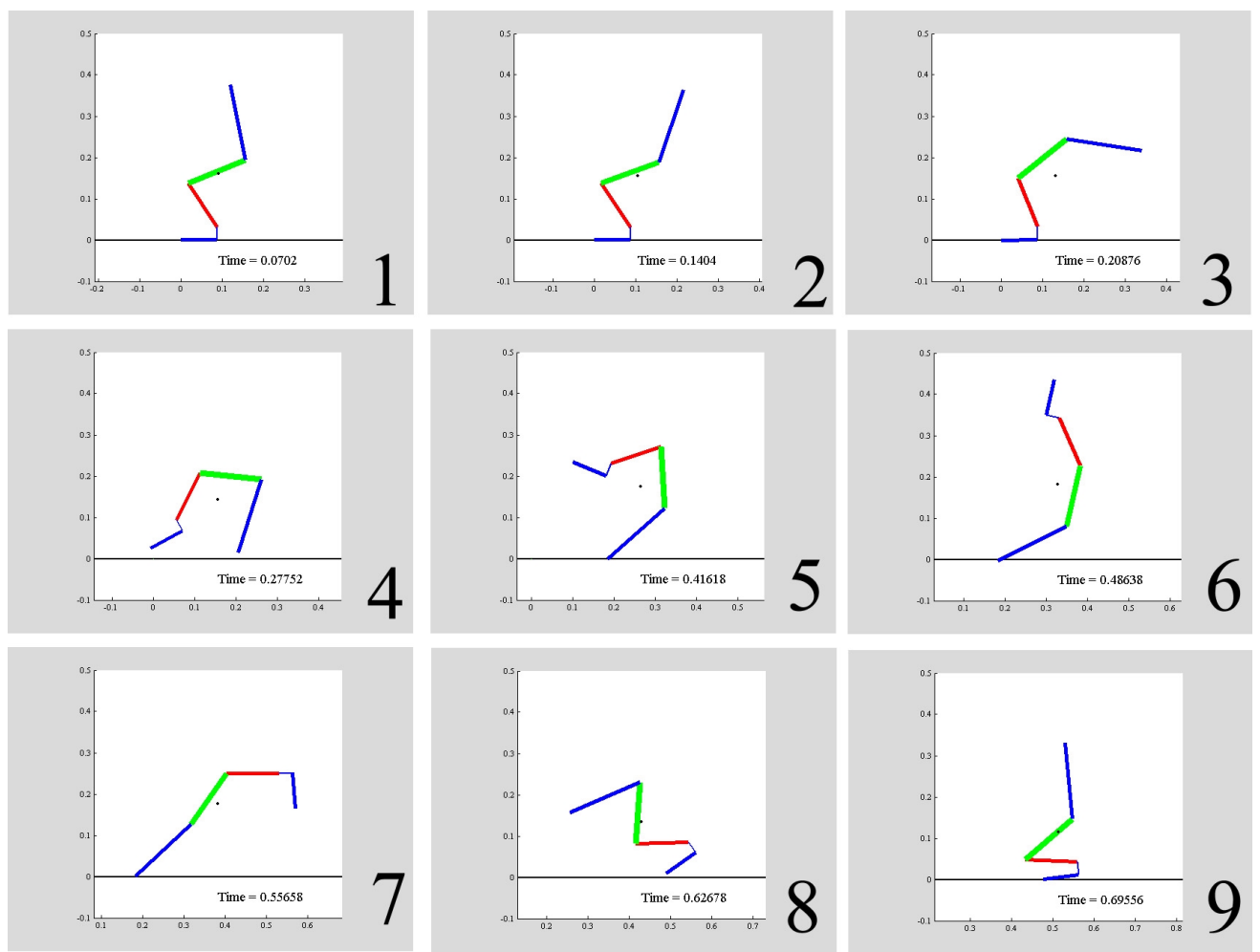

Fig. 4. Stick animation of back handspring simulation
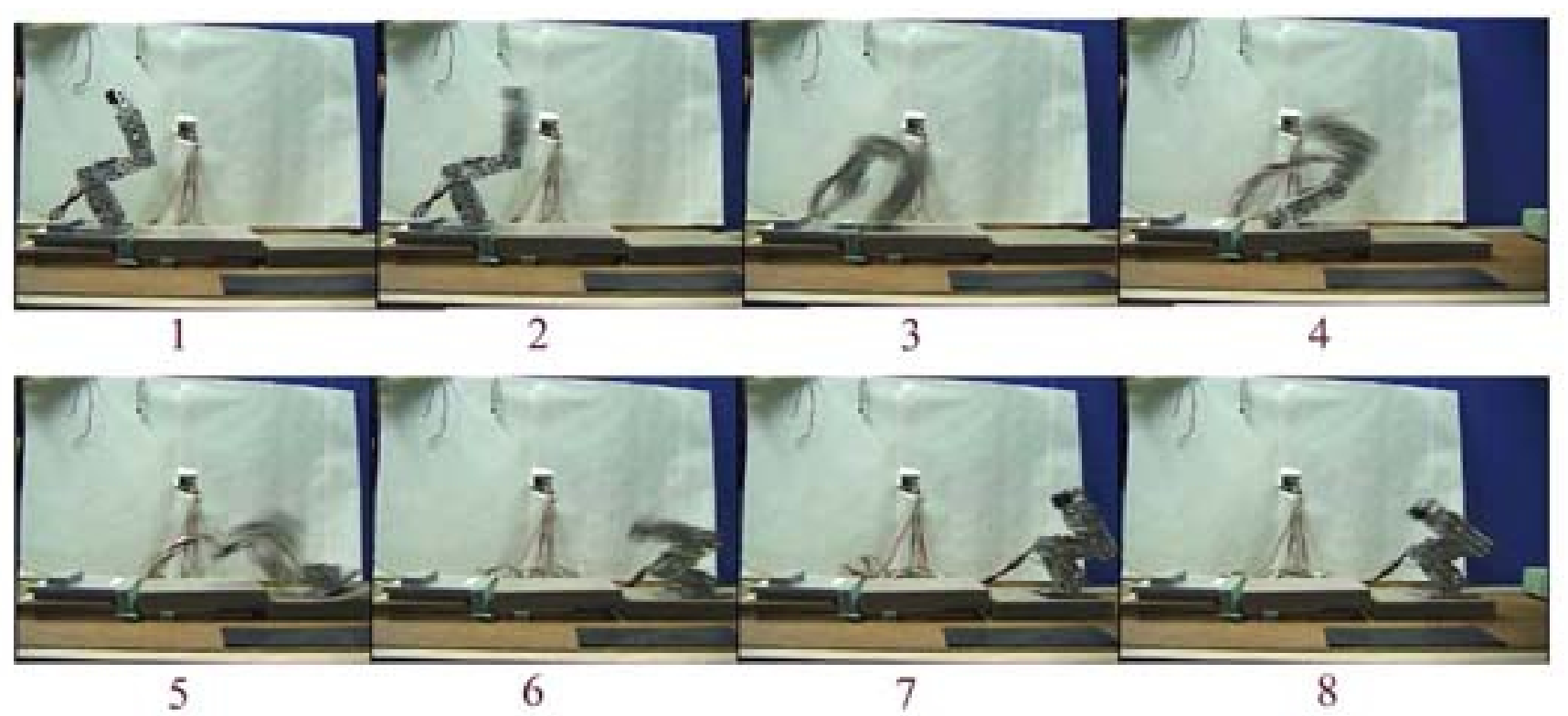

Fig. 5. Snap shots of back handspring experiment 


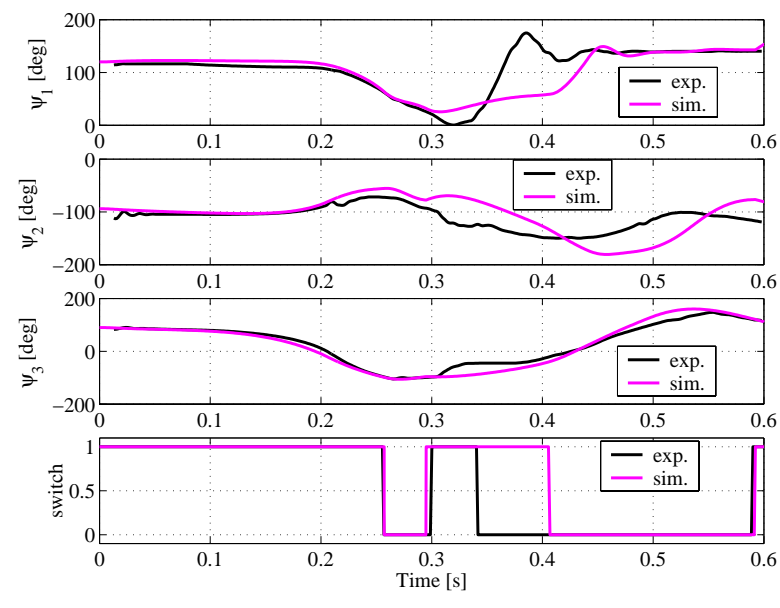

Fig. 6. Joint angles in simulation and experiment

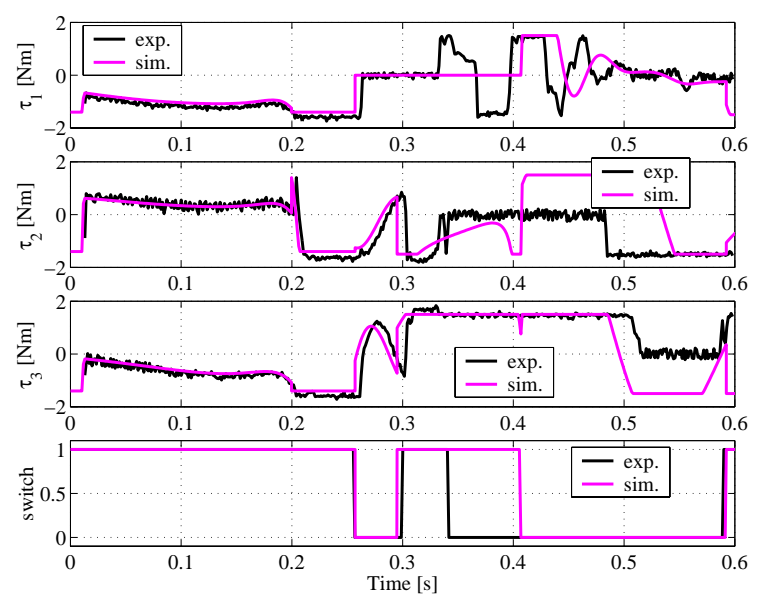

Fig. 7. Joint torques in simulation and experiment

control method that requires pre-planned reference trajectories. It was composed of task-specific target dynamics and its model matching. The use of global physical quantities such as center of mass, or angular momentum allowed even simple target dynamics to generate complex gymnastic motions of multi-body system. The effectiveness of the controller was confirmed via simulations and experiments of back handspring.

To the best of our knowledge, it is the first time that back handspring is performed by real multi-link robot. In simulation, we have also succeed in other interesting motion such as hopping, somersault. The somersault control, however, needs more joint torques than back handspring and not applicable to the current machine.

Since the model matching controller is working well, the remained important task is a systematic design of target dynamics. We think the key is the analysis and control of intermediate flight phase, which are not provided in this paper. If the lift-off configuration lies in some accessible region and there are enough flight time, we can steer the posture to some desired region until the next touchdown.
This is known as aerial attitude control problem with nonholonomic constraint, discussed in [12] [13] [14] [15]. Solution of this problem will leads to the systematic design of the control parameters of target dynamics at stance phase as well.

In this context, re-design of controlled variables is also important task because the robot has three control inputs, hence the maximum number of the target dynamics that can be followed independently is three. For example, instead of the position of $x_{g}$, we can introduce the target dynamics about configuration of the robot because the configuration at lift-off strongly affects on the aerial motion.

We believe more difficult task such as successive back handspring can be realized in the near future.

\section{ACKNOWLEDGEMENT}

The authors would give thanks to M. Suzuki for his great help in fabricating the hardware.

\section{REFERENCES}

[1] M. Raibert, Legged Robots That Balance. MIT Press, 1985.

[2] J. Hodgins and M. Raibert, "Biped gymnastics," Int. J. Robotics Researchvol, vol. 9, no. 2.

[3] K. Arikawa and T. Mita, "Design of multi-dof jumping robot," in Proc. of IEEE ICRA, 2002, pp. 3992-3997.

[4] M. Spong, "The swing up control problem for the acrobot," IEEE Control system magazine, vol. 15, no. 1, pp. 49-55, 1995.

[5] M. Yamakita, T. Yonemura, Y. Michitsuji, and Z. Luo, "Stabilization of acrobot in upright position on a horizontal bar," in Proc. of IEEE ICRA, 2002, pp. 3093-3098.

[6] J. Nakanishi, T. Fukuda, and D. Koditschek, "A brachiating robot controller," IEEE Trans. on Robotics and Automation, vol. 16, no. 2, pp. 109-123, 2000.

[7] M. Yamakita, M. Kishikawa, and T. Sadahiro, "Motion control for robust landing of acrobat robot (smb)," in Proc. of IEEE/RSJ IROS, 2003, pp. 1141-1146.

[8] M. Berkemeier and R. Fearing, "Tracking fast inverted trajectories of the underactuated acrobot," IEEE Trans. on Robotics and Automation, vol. 15, no. 4, pp. 740-750, 1999.

[9] M. Miyazaki, M. Sampei, and M. Koga, "Control of the motion of an acroboto approaching a horizontal bar," Advanced Robotics, vol. 15 , no. 4 , pp. 467-480, 2001.

[10] D. T. Greenwood, Classical dynamics. Prentice-Hall, 1977.

[11] T. Ikeda, T. Tamura, and T. Mita, "Development and running control of a 3d leg robot," in Proc. of AMAM, 2000.

[12] J. Godhavn, A. Balluchi, L. Crawford, and S. Sastry, "Steering of a class of nonholonomic systems with drift terms," Automatica, vol. 35, pp. 837-847, 1999.

[13] M. Sampei, H. Kiyota, and M. Ishikawa, "Control strategies for mechanical systems with various constraints," in Proc. of IEEE SMC, 1999, pp. 158-167.

[14] T. Mita, S. Hyon, and T. Nam, "Analytical time optimal control solution for a two link planar acrobot with initial angular momentum," IEEE Trans. on Robotics and Automation, vol. 17, no. 3, pp. 361-366, 2001.

[15] S. Hyon and T. Emura, "Aerial posture control for $3 \mathrm{~d}$ biped running using compensator around yaw axis," in Proc. of IEEE ICRA, 2003, pp. $57-62$. 